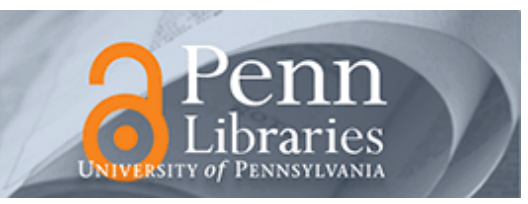

University of Pennsylvania

ScholarlyCommons

August 2002

\title{
Anomalous Mode Coupling in Guided-Wave Structures Containing Metamaterials with Negative Permittivity and Permeability
}

\author{
Andrea Alù \\ University of Pennsylvania, andreaal@seas.upenn.edu \\ Nader Engheta \\ University of Pennsylvania, engheta@seas.upenn.edu
}

Follow this and additional works at: https://repository.upenn.edu/ese_papers

\section{Recommended Citation \\ Andrea Alù and Nader Engheta, "Anomalous Mode Coupling in Guided-Wave Structures Containing Metamaterials with Negative Permittivity and Permeability", . August 2002.}

Copyright 2002 IEEE. Reprinted from Proceedings of the 2002 2nd IEEE Conference on Nanotechnology (IEEENANO 2002), pages 233-234.

Publisher URL: http://ieeexplore.ieee.org/xpl/tocresult.jsp?isNumber=22166\&page=3

This material is posted here with permission of the IEEE. Such permission of the IEEE does not in any way imply IEEE endorsement of any of the University of Pennsylvania's products or services. Internal or personal use of this material is permitted. However, permission to reprint/republish this material for advertising or promotional purposes or for creating new collective works for resale or redistribution must be obtained from the IEEE by writing to pubs-permissions@ieee.org. By choosing to view this document, you agree to all provisions of the copyright laws protecting it.

This paper is posted at ScholarlyCommons. https://repository.upenn.edu/ese_papers/59

For more information, please contact repository@pobox.upenn.edu. 


\title{
Anomalous Mode Coupling in Guided-Wave Structures Containing Metamaterials with Negative Permittivity and Permeability
}

\author{
Abstract \\ The peculiar characteristics of the mode coupling between standard dielectric waveguides and \\ waveguides formed by a material with negative index of refraction are presented in this talk. Some of our \\ theoretical results, describing the anti-directional (antiparallel) nature of such coupling, are shown for the \\ case of the two-parallel-slab geometry.

\section{Keywords} \\ coupled mode analysis, dielectric waveguides, microwave materials, permeability, permittivity, anomalous \\ mode coupling, anti-directional coupling nature, guided-wave structures, metamaterials, negative index of \\ refraction, negative permeability, negative permittivity, standard dielectric waveguides, two-parallel-slab \\ geometry

\section{Comments} \\ Copyright 2002 IEEE. Reprinted from Proceedings of the 2002 2nd IEEE Conference on Nanotechnology \\ (IEEE-NANO 2002), pages 233-234. \\ Publisher URL: http://ieeexplore.ieee.org/xpl/tocresult.jsp?isNumber=22166\&page=3 \\ This material is posted here with permission of the IEEE. Such permission of the IEEE does not in any way \\ imply IEEE endorsement of any of the University of Pennsylvania's products or services. Internal or \\ personal use of this material is permitted. However, permission to reprint/republish this material for \\ advertising or promotional purposes or for creating new collective works for resale or redistribution must \\ be obtained from the IEEE by writing to pubs-permissions@ieee.org. By choosing to view this document, \\ you agree to all provisions of the copyright laws protecting it.
}




\title{
Anomalous Mode Coupling in Guided-Wave Structures Containing Metamaterials with Negative Permittivity and Permeability
}

\author{
Andrea Alu' and Nader Engheta \\ University of Pennsylvania, Department of Electrical and Systems Engineering, \\ Philadelphia, Pennsylvania 19104-6390, U.S.A., Tel: +1-215-898-9777, Fax: +1-215-573-2068 \\ E-mail: andreal@ee.upenn.edu, engheta@ee.upenn.edu \\ URL: http://www.ee.upenn.edu/ engheta/
}

\begin{abstract}
The peculiar characteristics of the mode coupling between standard dielectric waveguides and waveguides formed by a material with negative index of refraction are presented in this talk. Some of our theoretical results, describing the anti-directional (antiparallel) nature of such coupling, are shown for the case of the two-parallelslab geometry.
\end{abstract}

\section{StATEMENT OF THE PROBLEM}

The concept of composite materials possessing negative permittivity and permeability at certain frequencies has recently attained considerable attention and interest [1]. The original idea for such media dates back to 1967 when Veselago theoretically studied time-harmonic monochromatic plane wave propagation in a material whose permittivity and permeability were assumed to be simultaneously negative [2]. Shelby, Smith, and Schultz recently constructed such a composite medium for the microwave regime, and experimentally showed the presence of anomalous refraction in this medium [3]. It is also interesting to note that the previous theoretical study of electromagnetic wave dispersion properties of the omega media using the circuit-model approach had also revealed the possibility of having negative effective permittivity and negative effective permeability in omega media for certain range of frequencies [4]. Recently as a potential application of these metamaterials, an idea was theoretically introduced for compact cavity resonators, in which a combination of a slab of conventional material and a slab of metamaterial with negative permittivity and permeability was inserted [5].

Metamaterials with negative permittivity and permeability, which have been named by several terminologies such as "double-negative" (DNG) media [6], "backward" (BW) media [7], left-handed media [1], [2], [3], [8], and negative-index media (NIM) [8], conceptually possess interesting electromagnetic features such as anomalous refraction. Moreover, when slabs of these materials are placed next to layers of conventional materials with positive real permittivity and permeability (which, in analogy with $\mathrm{DNG}$, can be called doublepositive (DPS) media), the combined structures may exhibit even more interesting properties, unique to the paired layers of DNG-DPS materials. For instance, a pair of DNG-DPS layers has been shown theoretically to provide phase conjugation/compensation [5].

In the present work, we study the electromagnetic mode coupling between layers of DNG and DPS materials. In order to understand the physics behind the phenomenon, we analyze the two-parallel-slab geometry (see Fig. 1), first using a rigorous exact approach and then using the perturbation theory for when the slab waveguides are not too close to each other. Our analysis shows that the mode coupling between DNG and DPS layers exhibits anomalous characteristics for what concerns the direction of power coupling. This effect is related to the fact that in a DNG slab the direction of the real part of the Poynting vector of a guided mode is antiparallel with the direction of phase flow of that mode, contrary to the case of guided modes in a standard DPS layer. Boundary conditions enforce the phase flow direction to be the same in the two slabs, and thus when a DNG slab is in proximity of a parallel slab made of a conventional material (i.e., a DPS medium), the power coupling is anti-directional (i.e., antiparallel).

In this talk, we will first give a brief review of electromagnetic properties of DNG media and their waveguiding properties, and then will present the results of our theoretical studies on mode coupling. We will then mention physical intuitions and insights into our findings.

\section{REFERENCES}

[1] D. R. Smith, W. J. Padilla, D. C. Vier, S. C. NematNasser, and S. Schultz, "Composite medium with simultaneously negative permeability and permittivity," Phys. Rev. Lett., vol. 84, no. 18, pp. 4184-4187, 1 May 2000 . 
[2] V. G. Veselago, "The electrodynamics of substances with simultaneously negative values of and "Soviet Physics Uspekhi, vol. 10, no. 4, pp. 509-514, 1968. (In Russian, Usp. Fiz. Nauk, vol. 92, pp. 517-526, 1967).

[3] R. A. Shelby D. R. Smith, and S. Schultz, "Experimental verification of a negative index of refraction", Science, vol. 292 , no. 5514 , pp. $77-79,6$ April 2001.

[4] M. M. I. Saadoun and N. Engheta, "Theoretical study of electromagnetic properties of non-local omega media" chapter 15 in Progress in Electromagnetic Research (PIER) Monograph series, vol. 9, A. Priou, (Guest Editor), 1994, pp. 351-397.

[5] N. Engheta, "An idea for thin subwavelength cavity resonators using metamaterials with negative permittivity and permeability," IEEE Antennas and Wireless Propagation Letters, Vol. 1, 2002, in press.

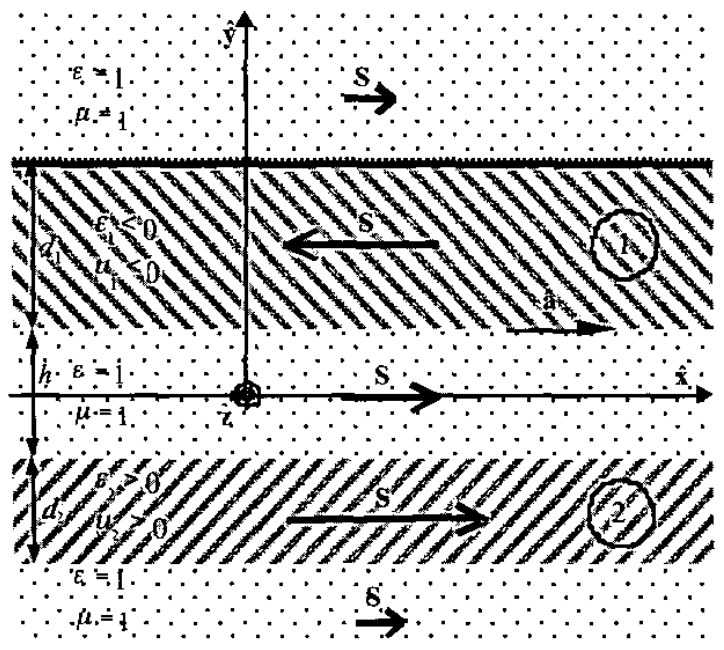

Fig.1 - Geometry of the structure
[6] R. W. Ziolkowski and E. Heyman, "Wave propagation in media having negative permittivity and permeability," Phys. Rev. E., vol. 64, no. 5, 056625, October 2001

[7] I. V. Lindell, S. A. Tretyakov, K. I. Nikoskinen, and S. Ilvonen, "BW media - Media with negative parameters, capable of supporting backward waves," Microwave and Optical Technology Letters, Vol. 31, No. 2, pp. 129133, October 2001

[8] R. A. Shelby, D. R. Smith, S. C. Nemat-Nasser, and S. Schultz, "Microwave transmission through a twodimensional, isotropic, left-handed metamaterials," Applied Physics Letters, Vol. 78, No. 4, pp. 489.491, 2001.

[9] P. M. Valanju, R. M. Walser, and A. P. Valanju, "Wave refraction in negative-index media: Always positive and very inhomogeneous," Physical Review Letters, Vol. 88, No. $18,012220,2002$. 